\title{
Défis et enjeux de la gouvernance locale au Cameroun à l'ère de la décentralisation : cas de la ville de Garoua
}

\section{PETNGA NYAMEN Simon Pierre}

PhD en Géographie, Téléphone : (+237) 697259721, Université de Ngaoundéré, École Normale Supérieure de Bertoua,

Département de Géographie, BP : 652 - Bertoua, Cameroun, pnspierre@gmail.com.

\begin{abstract}
Résumé
Le Décret N²007/115 du 23 avril 2007 portant création de nouveaux Arrondissements au sein de certains Départements du Cameroun traduit a priori la volonté du Gouvernement d'insuffler une dynamique nouvelle à son processus de décentralisation. À partir du cas de la ville de Garoua, ce travail traite des défis et enjeux de la gouvernance locale dans un contexte d'accélération du processus de décentralisation en vue d'un développement maitrisé. Pour ce faire, des échanges avec vingt-six informateurs issus de l'administration publique et privée, douze chefs de quartier et trente des plus anciens habitants de Garoua ont été mené. En plus, on a eu recours à une centaine d'informateurs et guides, qui ont permis de caractériser trois cent cinquante-cinq marqueurs spatiaux de la dynamique urbaine de la localité. Les résultats de cette étude révèlent que les défis et enjeux actuels de la gouvernance locale sont de trois ordres : règlementaire, financier et fonctionnel. Pour ce qui est du premier ordre, le problème des villes camerounaises, est le non-respect de la règlementation en vigueur, et surtout l'omniprésence de la corruption. Le deuxième ordre est celui de l'incapacité des municipalités à collecter les recettes ce qui ne limite leur investissement qu'à des ouvrages de très faible impact social et économique. Sur le plan fonctionnel, la décentralisation a favorisé la multiplication d'acteurs aux aspirations très souvent divergentes, mais aussi une confusion voire une ignorance des rôles. Au terme de cette étude, avec l'adoption de comportements légaux, il est recommandé aux administrations locales de s'ouvrir au Système d'Information Géographique (SIG) qui dispose des méthodes, techniques et outils permettant de gérer efficacement la donnée spatiale et par conséquent le territoire. De plus, le processus de création de ce système utilisé pour la collecte, le stockage, l'analyse, la modélisation, la gestion, l'affichage et la représentation de l'information spatiale, est une excellente aubaine pour la mise en œuvre d'un cadre de concertation qui intègre à différentes échelles, la représentativité, les compétences et les objectifs respectifs de toutes les parties prenantes à la gouvernance locale.
\end{abstract}

Mots clés : Gouvernance locale, Décentralisation, Développement maîtrisé, SIG, Garoua.

Received: $14 / 02 / 2020$
Accepted: $23 / 09 / 2020$
DOI: https//dx.doi.org/10.4314/jcas.v16i2.7
C The Authors. This work is licensed under the Creative Commons Attribution 4.0 International Licence.




\begin{abstract}
Decree N²007/115 of April 23, 2007 creating new subdivisions within some Divisions of Cameroon demonstrates the will of the Government to improve its decentralization process. Based on the case of the city of Garoua, this work deals with the challenges and issues of local governance, in a context of accelerating the decentralization process with a view to controlled development. To this end, exchanges with twenty-six informants from the public and private administration, twelve chiefdom leaders and thirty of the oldest inhabitants of Garoua were conducted. In addition, about one hundred informants and guides were used, who allowed to characterize three hundred and fifty-five spatial markers of the dynamics of the city. The results revealed that the current challenges and issues of local governance are threefold: regulatory, financial and functional. As for the first, the problem of Cameroonian cities is the non-compliance with the regulations in force, and especially the pervasiveness of corruption. The second order is the inability of municipalities to collect revenue, which limits their investment to works of very low social and economic impact. From a functional point of view, decentralization has favored the multiplication of actors with very divergent aspirations, but also confusion and ignorance of roles. Based on these results, we recommend that local governments open up to Geographic Information System (GIS), which has the methods, techniques and tools to effectively better manage the spatial data and consequently the territory. In addition, the process of creating this system, used for the collection, storage, analysis, modelling, management, display and representation of spatial information, is an important opportunity towards the implementation of a consultation framework that integrates, at different levels, the representativeness, skills and objectives of all stakeholders of local governance on the field.
\end{abstract}

Keywords: Local Governance, Decentralization, Controlled Development, GIS, Garoua. 


\section{Introduction}

Avec un taux d'urbanisation de l'ordre de 52,0\% (BUCREP, 2010), le Cameroun s'est hissé au niveau des pays les plus urbanisés d'Afrique francophone au Sud du Sahara. En inscrivant le développement urbain dans les priorités de son Document de Stratégie pour la Croissance et l'Emploi (DSCE) ${ }^{1}$ et en y consacrant plus d'un tiers de son second Contrat de Désendettement Développement (C2D) ${ }^{2}$, le Gouvernement du Cameroun marque ainsi son profond intérêt pour le devenir de ses espaces urbains. Cet intérêt a conduit à une reformulation des orientations nationales en matière de gestion des villes, se traduisant par la recherche de nouveaux flux de financements nationaux et internationaux destinés à améliorer la gouvernance urbaine d'une part, et par la mise en œuvre du processus de décentralisation, qui doit accroitre la participation des populations locales à l'amélioration de leurs conditions de vie d'autre part (Petnga Nyamen, 2018).

La récente organisation administrative du territoire national s'explique donc a priori par la volonté gouvernementale d'insuffler une nouvelle dynamique à son processus de décentralisation. Définie comme étant le transfert de certaines compétences et des moyens qui étaient jusque-là dévolus à l'État aux Collectivités Territoriales
Décentralisées (CTD), la décentralisation a redonné un pouvoir d'initiative aux acteurs locaux tout en redistribuant les responsabilités administratives (Pumain, 2008). Parmi les compétences transférées aux CTD camerounaises, il y a la gestion urbaine qui, selon Elong M'bassi (2001) cité par Ouedraogo (2010), consiste en l'amélioration des infrastructures et des services urbains afin d'accroitre le développement de la ville et par conséquent, améliorer les conditions de vie des citadins (Petnga Nyamen, 2018). Ainsi, la problématique centrale de ce travail interroge les défis et les enjeux de la gouvernance locale au Cameroun dans un contexte d'accélération du processus de décentralisation en vue d'une émergence maîtrisée.

\section{Approche méthodologique adoptée}

Cette étude a pour référent spatial, la ville de Garoua telle que définie par le décret n²008/020 du 17 janvier 2008, portant création de la Communauté Urbaine de Garoua, et qui fixe le ressort territorial de la ville de Garoua à celui de ses trois Communes d'Arrondissement, créées l'année précédente. Ainsi, la ville de Garoua est située entre $8^{\circ} 25$ et $10^{\circ}$ de latitude Nord, $13^{\circ} 30$ et $14^{\circ} 25$ de longitude Est. Elle est limitée au Nord par les Communes de Gashiga, Demsa, de Pitoa et les massifs du Mont Tinguelin, au Sud et à l'Est par la Commune de Tchéboa (Figure 1).

\footnotetext{
${ }^{1}$ Couvrant la période 2010-2020 et centré sur la croissance économique, la création d'emplois formels et la réduction de la pauvreté au Cameroun, le DSCE porte une attention particulière à l'intégration régionale et à l'amélioration de la gouvernance, notamment par la lutte contre la corruption, la transparence dans l'attribution et l'exécution des marchés publics, et l'amélioration du climat des affaires à l'horizon 2035, afin de réaliser les Objectifs du Millénaire pour le Développement (OMD) dans leur ensemble (Edoa, 2014).

${ }^{2}$ C'est pour le Cameroun le plus important programme d'annulation et de reconversion de sa dette extérieure. En pratique, l’État camerounais continue d'honorer sa dette mais, aussitôt le remboursement constaté, la France reverse la somme correspondante sous forme de dons pour qu'elle soit affectée à des programmes de lutte contre la pauvreté sélectionnés d'un commun accord avec le Cameroun (AFD, 2015).
} 
REVUE DE L'ACADEMIE DES SCIENCES DU CAMEROUN Vol. 16 No. 2 (Sept 2020)

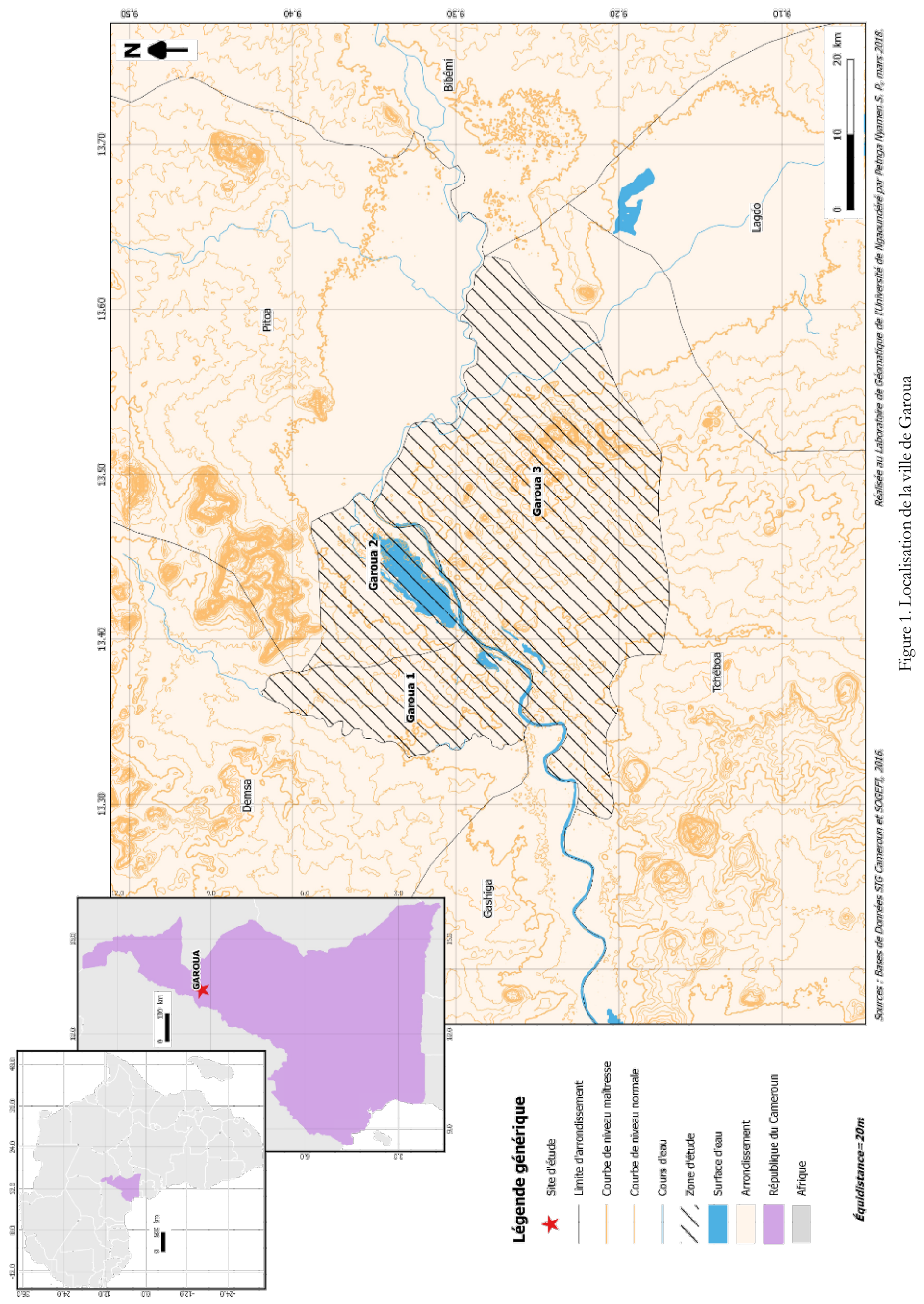


L'approche méthodologique adoptée pour mener à bien cette recherche est centrée sur l'analyse de la perspective spatiale et temporelle de la gestion locale, qui découle d'une expérience ancrée sur le terrain : un espace vécu, observé et interprété. Cette démarche ne repose pas sur une simple procédure d'enquête unique et linéaire, mais souscrit à une démarche qualitative et interprétative.

Pour la construction de la base du sondage destinée à l'analyse du mode de gestion urbaine de Garoua, la méthode d'échantillonnage non probabiliste a été privilégiée. Cette dernière consiste à identifier les informateurs pertinents, grâce à une technique fondée sur une démarche inductive qui permet de recruter des personnes, par l'intermédiaire des connaissances, à qui on demande de désigner d'autres personnes correspondant aux critères retenus notamment: la fonction sociale, l'âge, le sexe (de par les traditions africaines, les hommes sont les principaux concernés par les questions foncières, d'habitat et d'occupation des sols), et la représentativité des enquêtés. Ainsi, on a pu entrer en contact avec soixante-huit (68) potentiels informateurs, dont vingt-six (26) issus de divers secteurs de l'administration publique et privée, douze (12) chefs de $3^{\text {ème }}$ degré et trente (30) autochtones, c'est-à-dire des personnes parmi les plus anciens habitants de la ville. En plus, on a eu recours à cent (100) informateurs et guides, qui ont permis de caractériser trois cent cinquante-cinq (355) marqueurs spatiaux de la dynamique de la ville.

\section{Matériels et outils}

La recherche documentaire, a été menée auprès de la Communauté Urbaine de Garoua (CUG), des Communes d'Arrondissements de la ville, de la représentation régionale du Programme National de Développement Participatif (PNDP), dans les archives de l'archidiocèse de Garoua, on a aussi exploité les articles et thèmes traitant de la ville de Garoua et des migrations dans le Nord Cameroun.

Les entretiens semi-dirigés ont été particulièrement adéquats dans ce contexte car ils ont permis à la fois de s'assurer que l'on couvre les éléments importants pour répondre aux questions de recherche, et que l'on donne aux répondants l'opportunité d'apporter leurs propres idées et d'exprimer leurs pensées par rapport aux thèmes abordés. Ils permettent donc de découvrir le sens et les finalités que les acteurs associent à leur situation et à leurs actions. C'est dire que le processus de recherche sur le terrain a été dynamique et interactif en donnant un contenu scientifique aux propos des informateurs.

L'instrument de collecte des données est issu des entretiens qualitatifs menés conjointement à la collecte documentaire. Il a été question d'interpréter et de discuter les résultats des enquêtes orales, de les confronter à d'autres sources (écrites et cartographiques) afin d'en dégager les points de convergence et de divergence. L'analyse a consisté au traitement des données, à leur analyse transversale et verticale. Il s'est agi d'une entreprise dynamique, en constante progression, alimentée en permanence par le travail sur le terrain.

En ce qui concerne le rendu cartographique, on a eu recourt à des images satellites de haute résolution de Garoua obtenues via Mapbox, qu'on a intégré à une Base de Données Système d'Information Géographique (SIG) de la ville créée dans le cadre des travaux du Laboratoire de Géomatique de l'Université de Ngaoundéré. La méthode utilisée pour la création de la Base de Données SIG de la CUG a nécessité des matériels et outils de la géomatique spécifiques.

$\checkmark \quad$ Des images satellites et Radar, obtenues à partir des sources de données disponibles et téléchargées gratuitement sur internet; 
$\checkmark \quad$ Des cartes topographiques: après géoréférencement, elles ont permis de confronter en laboratoire les résultats des opérations automatiques;

$\checkmark \quad$ Du matériel et outils informatiques, les applications opensource et/ou en libre accès sur internet ont été privilégiées notamment: GoogleEarthPro ; El-shayal ; BaseCamp ; Calc d'OpenOffice, QGIS et GPS Essentials.

Comme pour la réalisation de tout projet cartographique à l'aide d'un logiciel SIG, celle de Garoua comprend les phases suivantes :

$\checkmark \quad$ Acquisition et exploitation des données de base. Elle a été réalisé auprès des autorités administratives, des sectoriels, des chefs traditionnels et des données spatiales en libre accès sur internet;

$\checkmark \quad$ De la vérification - terrain, qui a consisté en la confrontation des données de base à la réalité du site d'étude, afin de sélectionner et puis valider les informations utiles à la réalisation du projet ; $\checkmark \quad$ Du laboratoire à la carte, c'est l'étape consistant à la création des différentes couches du SIG à l'aide du logiciel approprié qui est QGIS dans le cas de la présente étude ;

$\checkmark \quad$ De la validation de la Base de Données. Elle devra se faite au cours d'une séance de travail avec tous les acteurs impliqués dans la gestion urbaine pour la présentation et la validation de la BD.

\section{Résultats}

1. Gouvernance locale, des domaines d'intervention variés mais financés en grande partie par l'État

Composée des Communes d'Arrondissement de Garoua $1^{\text {er }}, 2^{\text {ème }}$ et $3^{\text {ème }}$, la CUG est actuellement dotée d'un paysage urbain marqué principalement par des zones d'occupation spontanée et des secteurs planifiés à l'avance. Tout au long de son histoire et en fonction des besoins de la population, différentes administrations locales ont intervenu sur l'espace urbain, en l'équipant de plusieurs infrastructures, couvrant les domaines de l'éducation, du social, du commerce, de la santé des personnes, des voies de communication, de l'approvisionnement en eau, de la santé animale et de l'administration. L'analyse de ces réalisations a permis de déterminer les secteurs que les différentes administrations de Garoua ont estimés prioritaires pour le développement local. Il ressort en que l'éducation est le principal (Figure 2).

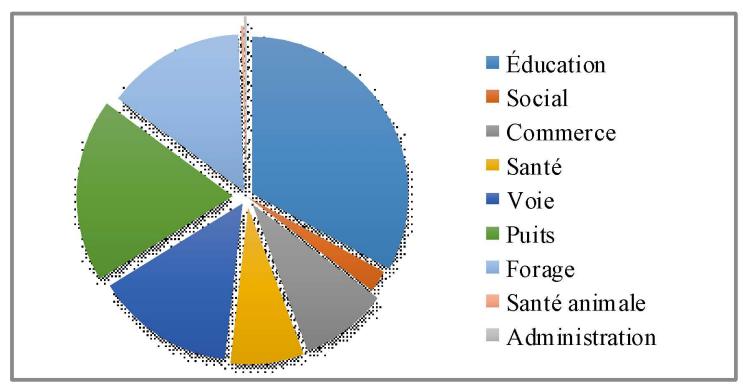

Source : Enquêtes de terrain, avril 2016.

Figure 2. Répartition des équipements urbains de Garoua par domaine d'intervention

Pour ce qui est du financement de ces différentes interventions, il ressort des enquêtes de terrain que, pour l'ensemble des Communes de cette ville l'État en est la principale source (Figure 3).

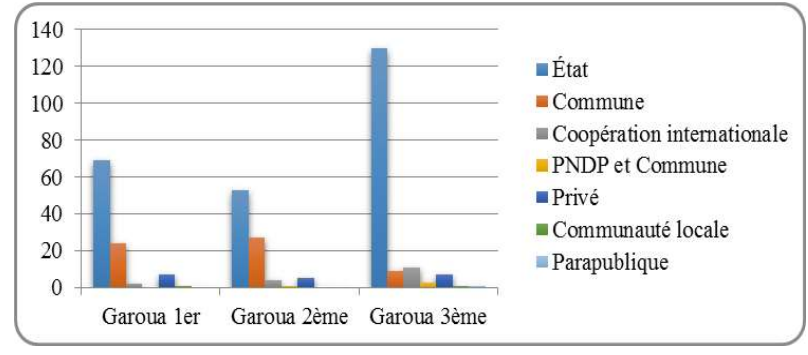

Source: Enquêtes de terrain, avril 2016.

Figure 3. Répartition des principales sources de financement des différents équipements de la ville de Garoua par Commune

Néanmoins, sans sous-estimer la contribution de l'État du Cameroun au développement local, il est important de relever que dans le contexte actuel de décentralisation, ce n'est plus son rôle. L'État doit plutôt créer les conditions économiques, politiques et sociales favorables aux investissements privés à l'échelle locale. C'est donc aux CTD que revient la responsabilité de 
trouver les sources de financement de leur développement, par exemple, en signant des conventions de partenariat avec les municipalités des pays développés pour des partages d'expériences, ou avec les institutions financières nationales et internationales d'une part, et les entreprises privées d'autre part pour l'équipement de la ville et sa rentabilisation. Pour y parvenir, les CTD doivent avant tout ouvrer pour une meilleure gestion foncière.

\section{Quels outils pour une meilleure gestion urbaine à l'ère de la décentralisation ?}

L'un des plus importants défis à relever, pour une gouvernance locale plus efficace, est la maîtrise du territoire, sans laquelle aucune gestion foncière efficace n'est possible. En l'absence de politiques de gestion anticipative (Tchotsoua et Bonvallot,
2000 ; Tchotsoua et al., 2001 ; Petnga Nyamen, 2018), du territoire de la ville, les différentes interventions des acteurs locaux aboutissent au désordre, à l'insalubrité, bref constituent un sérieux frein à l'émergence socio-économique des communautés locales. Or, les progrès réalisés dans les différents champs de la géomatique ouvrent de nouvelles possibilités pour la maîtrise des territoires quel qu'ils soient. Grâce aux moyens de positionnement assisté par satellite notamment le GPS d'une part, et aux outils de cartographie moderne communément appelés SIG d'autre part, la carte n'est plus une arme au service exclusif des puissants. Le SIG permettant en effet, d'acquérir l'information spatiale, d'interroger et explorer la Base de Données. L'objectif ici est de mieux organiser les informations spatiales dans une Base de Données SIG (Figure 4) afin d'en faciliter la gestion et par conséquent la prise de décision.

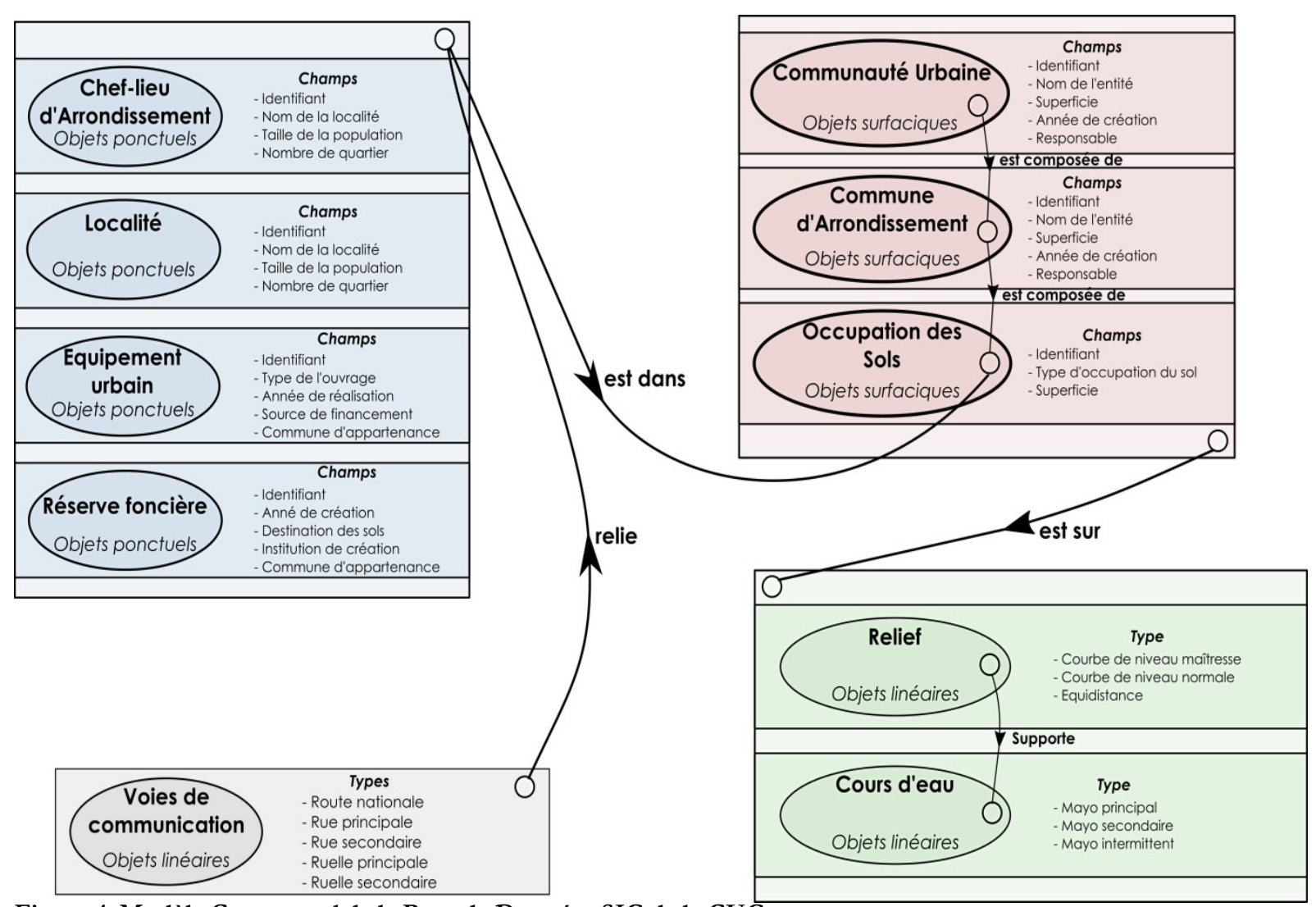

Figure 4. Modèle Conceptuel de la Base de Données SIG de la CUG

Il est important de signaler ici que le résultat obtenu (Photo 1) n'a pas force de loi, il a été réalisé dans le cadre d'un exercice scientifique. La méthode ci-dessus présentée, peut être utilisée dans un cadre légal comme outil d'aide à la décision pour la maitrise de l'emprise spatiale des territoires urbains. 


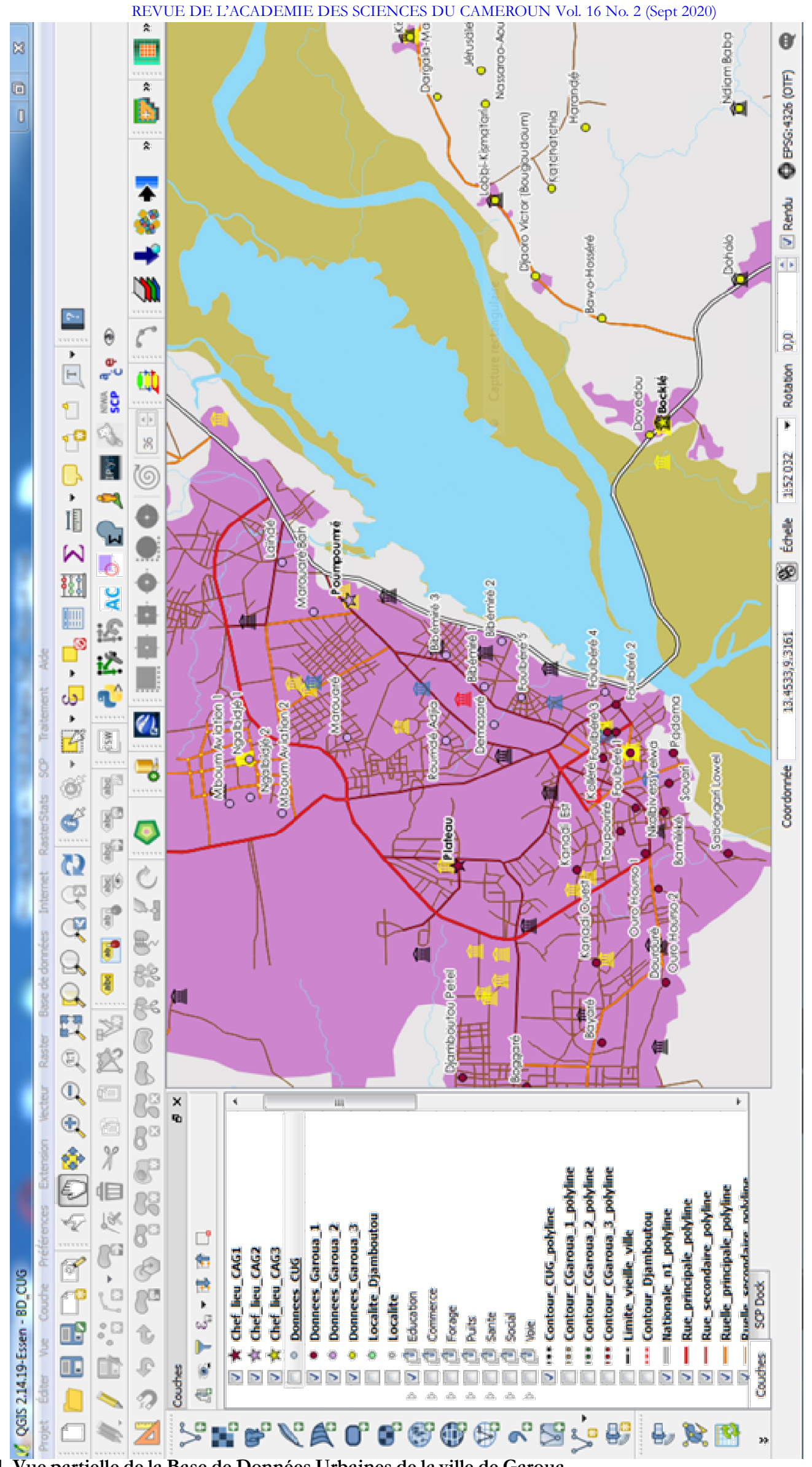

Photo 1. Vue partielle de la Base de Données Urbaines de la ville de Garoua 
Au-delà de l'intérêt scientifique et méthodologique certain de ce travail, il est appliqué parce qu'il pose les jalons de la planification que nul ne peut prétendre à réaliser sans une bonne maitrise du territoire (Petnga Nyamen et al., 2015 ; Petnga Nyamen, 2018).

\section{Enjeux d'un développement urbain maîtrisé au Cameroun}

\subsection{Sur le plan règlementaire}

Pour ONU-Habitat (2014), "les récentes mesures de décentralisation ont ajouté aux difficultés de la gouvernance, les autorités municipales restant incapables de se préoccuper de la majorité pauvre des populations urbaines en raison de leur inefficacité, de la bureaucratisation à outrance ainsi que de la corruption et du népotisme qui, de manière directe ou indirecte, donnent la préférence aux plus fortunés et à ceux qui sont politiquement bien introduits ». Allant dans le sens du constat de l'institution onusienne, Assako Assako et Njouonang Djomo (2015) soutiennent que le principal problème des villes camerounaises, est le non-respect de la règlementation en vigueur, et surtout l'omniprésence de la corruption. Une expression latine ne dit-elle pas "dura lex, ced lex », pour traduire le fait que la rigueur de la loi ne saurait s'ériger en circonstances atténuantes de sa violation. En effet, la difficulté d'application de la loi n’induit pas forcement l'impossibilité de la respecter. La conséquence du non-respect des principes et des règles de l'urbanisme dans la dynamique spatiale est la forte exposition des citadins aux aléas de la nature à l'instar des inondations (Planche photographique 1).

Au-delà de toutes les insuffisances et/ou contradictions qu'on pourrait être amené à relever au cours de la lecture des textes de lois relatifs à la décentralisation et à l'urbanisme au Cameroun, il ne faut pas perdre de vue l'essentiel, qui est d'adopter des comportements légaux, susceptibles de participer à l'amélioration des conditions de vie de l'ensemble, ou du moins, de la plus grande partie de la communauté locale. L'atteinte de cet

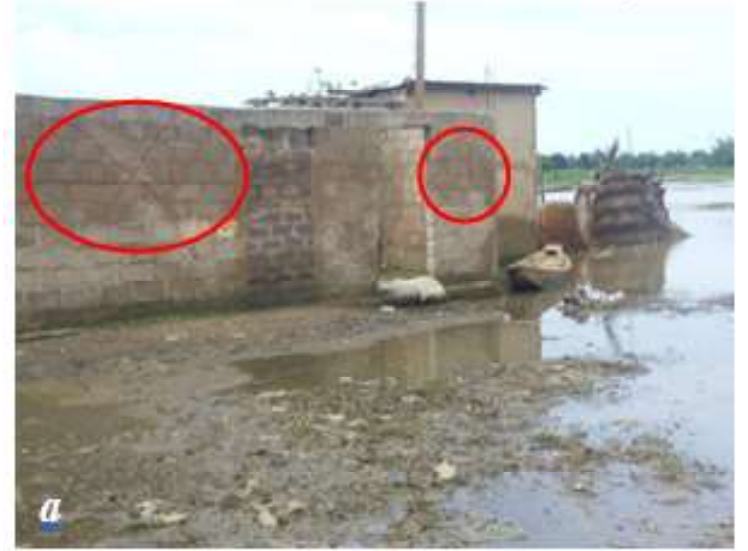

Longitude: 13,406420 ; Latitude: 09,308505. (C) Petnga Nyamen, 13/09/2012.

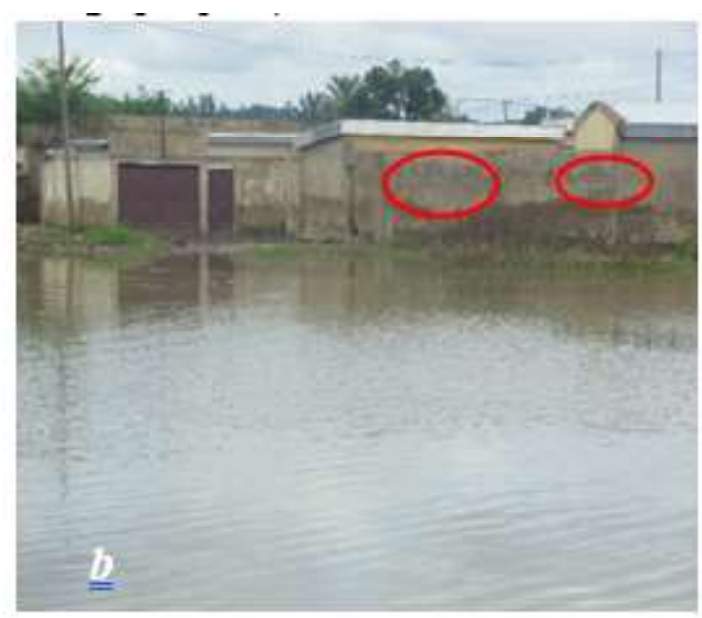

Longitude : 13,405554; Latitude : 09,306503.

(C) Petnga Nyamen, 14/09/2012

Planche photographique 1. (a) Des habitations exposées aux inondations à Bibémiré et (b) des maisons marquées par la Communauté Urbaine de Garoua

objectif passe nécessairement par l'équipement immobilier de la collectivité, ce que l'ONUHabitat (2014) appelle «une offre améliorée de services urbains intégrés».

\subsection{Sur les plans financier et fonctionnel}

Par ailleurs, l'incapacité des municipalités à «collecter les recettes qui leur permettraient de financer des services ", est un sérieux handicap au développement urbain. En dépit, de l'accélération de la décentralisation souhaitée par l'État, il continue d'être le principal financeur du développement local. Cet état des choses est un sérieux handicap, quand on sait que l'objectif de la décentralisation est de doter les collectivités d'une certaine autonomie, pour leur 
permettre d'atteindre les cimes de leur potentiel, et de participer, par conséquent, au développement du pays tout entier. Dans la quasitotalité des CTD du Cameroun, on constate que les recettes municipales sont extrêmement faibles, du fait de la non-diversification des sources de financement. Le résultat est que les collectivités fonctionnent exclusivement grâce aux Centimes Additionnels Communaux, ce qui ne limite leur investissement qu'à des ouvrages de très petite envergure. De plus, la faible implication des bailleurs de fonds nationaux, ou étrangers, ou même ceux des organisations de la société civile, couplée à l'absence quasi déconcertante des partenaires privés au développement, ne fait que réduire la capacité des collectivités locales à œuvrer pour l'amélioration des conditions de vie des citoyens.

En outre, la décentralisation a favorisé une multiplication d'acteurs locaux aux aspirations très souvent divergentes, mais aussi une confusion voire une ignorance des rôles, au plan institutionnel et surtout au plan de la pratique, ce qui constitue une entrave à certains projets de développement. À partir des données collectées à Garoua, il se distingue deux principaux types : les acteurs institutionnels dont un groupe opère à l'échelle nationale et l'autre à l'échelle locale ; les acteurs du secteur privé constitués d'une part des acteurs formels ou des associations, et des acteurs informels d'autre part. Pour ce qui est des rapports qu'ils entretiennent, à l'exception de quelques dysfonctionnements, il ressort que la collaboration entre les acteurs institutionnels et les acteurs du secteur privé peut être qualifiée de franche, d'autant plus que, les conflits en matière de gestion foncière sont rares entre ces acteurs. Par contre, pour ceux d'un même groupe, il existe une confusion de rôle du fait de la divergence des objectifs, du chevauchement des compétences et surtout de l'inexistence d'un cadre légal définissant clairement leur champ d'action respectif. Dans un tel contexte, les pouvoirs publics, rencontrent beaucoup de difficultés pour le contrôle des transactions foncières, d'où le constat désolant de leur démission. Cette situation a pour conséquence de laisser libre cours à la gestion coutumière des terrains, surtout pour des espaces où les chefferies traditionnelles ont toujours une influence sociale considérable.

\section{Discussion}

Qu'elles soient publiques, nationales, urbaines, rurales ou foncières, les politiques sont toujours des moyens, mieux, des instruments au service du développement des communautés humaines. En tant que politique publique, la décentralisation se doit de participer à la polarisation sociale et produire la cohésion nationale, par des procédures conduisant au consensus entre les acteurs qui gèrent la ville (Aldershot et al., 2000 ; Andersen et Van Kempen, 2001 ; Petnga Nyamen, 2018). Ainsi, il est indispensable qu'elle concilie la dimension législatrice, c'est-à-dire la production et le strict respect des lois, à la composante humaine conduite par les différentes administrations avec le numérique comme principal outil d'aide à la décision, afin que l'accélération du processus de décentralisation impulsée par le Chef de l'État, puisse réellement participer à l'émergence du Cameroun par l'amélioration des conditions de vie de tous les citoyens, puisque c'est la priorité.

\section{Conclusion}

À partir du cas de la ville de Garoua, l'objectif de cet article était d'analyser les défis et les enjeux de la gouvernance locale dans un contexte d'accélération du processus de décentralisation en vue d'un développement maîtrisé. Il en ressort que les administrations locales interviennent dans divers domaines de la vie sociale, bien que ces investissements sont en grande partie financés par l'État. Loin de sous-estimer la contribution de l'État au développement local, il est important 
de relever que dans le contexte actuel de décentralisation, ce n'est plus son rôle. En effet, c'est aux CTD que revient la responsabilité de trouver les sources de financement de leur développement. Pour y parvenir, les CTD doivent avant tout œuvrer pour une meilleure gestion foncière. Or, les progrès réalisés dans les différents champs de la géomatique ouvrent de nouvelles possibilités pour la maîtrise des territoires quel qu'ils soient. Étant donné qu'aujourd'hui, le SIG permet d'acquérir et stocker une grande masse de données géographiques, qui peuvent par la suite être interrogées ou exploitées par les différentes administrations locales pour une meilleure prise de décision. Il est important de relever ici que le processus de réalisation d'une Base de Données SIG urbaines est une merveilleuse aubaine de mettre en cohérence les desideratas de toutes les parties prenantes, afin d'arriver à un consensus sur la politique et les stratégies locales de gouvernance du territoire urbain.

\section{Recommandations}

La première recommandation formulée porte sur la formation des administrateurs locaux à l'utilisation et l'exploitation des produits de la géomatique comme outils d'aide à la décision. L'objectif est que toutes personnes en charge de la gestion des affaires publiques locales c'est-àdire les autorités traditionnelles, les magistrats municipaux et régionaux, soient capables d'analyser les dynamiques spatiales et infrastructurelles de leur territoire de compétences respectif sur un ordinateur, une tablette ou un smartphone.

La deuxième est la mise en place d'un cadre de concertation ouvert et franc entre tous les acteurs. Le rôle de cette plateforme d'échanges devra être la facilitation de la prise en compte des initiatives émanant des parties prenantes non étatiques, et surtout celles des populations défavorisées puisqu'elles sont les premières bénéficiaires des services de base. Placer la concertation au cœur des systèmes managériaux, politiques, économiques et sociaux, donne l'opportunité aux acteurs locaux de décider ensemble. De ce fait, les parties prenantes prennent l'engagement de partager les responsabilités qui en découlent et sont dès lors toutes gagnantes.

La troisième recommandation est un appel à une gestion urbaine prospective. Il est plus que jamais urgent pour l'administration locale, de s'impliquer dans la gestion des zones péri-urbaines, encore fortement rurale, par une bonne planification. Le laxisme ambiant, et le non-respect des lois et règlements de l'urbanisme, observés dans la conduite de certains projets de développement dans les centres urbains actuels, ne doivent pas se reproduire sur ces nouveaux fronts d'urbanisation. La figure 5 ci-dessous fait une présentation synthétique de ces recommandations.

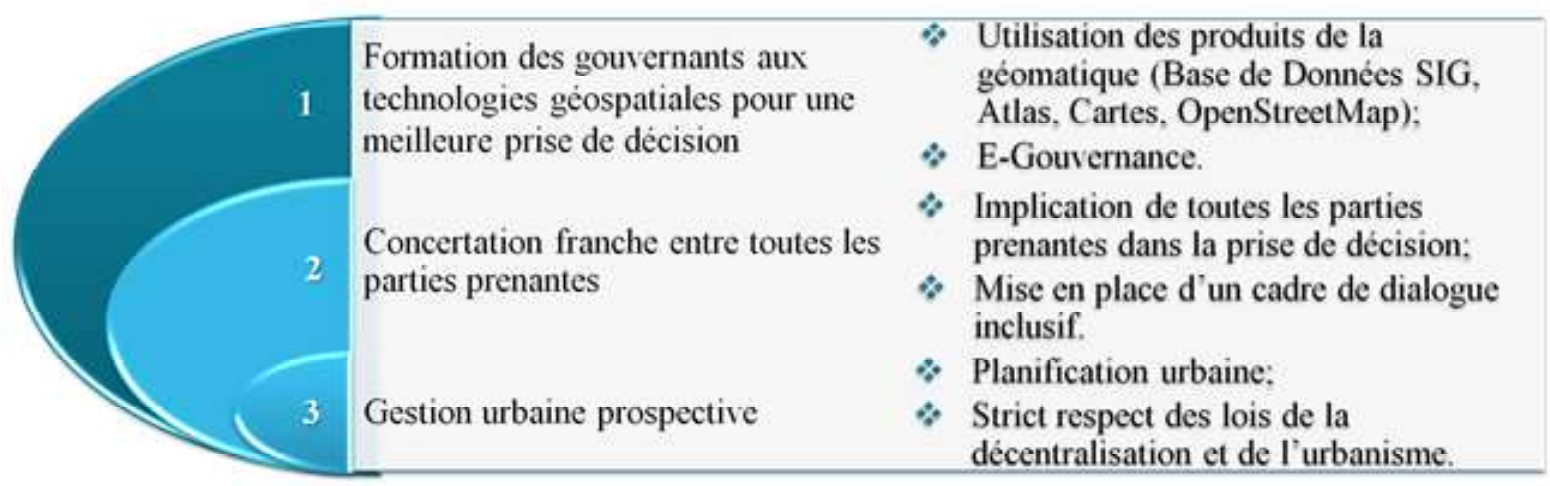

Figure 5. Schéma synthétique des recommandations 
Références bibliographiques

AFD, (2015). Outils et principes d'intervention de l'AFD. Consulté le Décembre 27, 2016, sur Agence Française de Développement: http:// www.afd.fr/home/pays/afrique/geo-afr/ cameroun/Strategie/outils-et-principes-dintervention-de-l-afd.

Aldershot, A., Maertens, C. et Vervaeke, M. (2000). La polarisation sociale des villes européennes. Economica.

Andersen, et Van Kempen, (Éds.) (2001). Governing european cities, social fragmentation, social exclusion and urban governance.

Assako Assako, R. J. et Njouonang Djomo, H. G. (2015). Curée foncière et stratégies d'accès à la terre dans la périphérie sud-ouest de Yaoundé. Syllabus - Revue scientifique interdisciplinaire de l'École Normale Supérieure - Série Lettres et sciences humaines, VI(1), 9-42.

BUCREP, (2010). La population du Cameroun en 2010. Rapport de présentation des résultats du $3^{\text {ème }}$ Recensement Général de la Population et de l'Habitat, Yaoundé-Cameroun, 9 pages.

Edoa, G. D. (2014). Optimisation de l'investissement public pour l'atteinte des objectifs. Conférence annuelle des responsables des services centraux et déconcentrés du MINEPAT, Yaoundé-Cameroun, 1-7.

Elong M'bassi, J. P. (2001). Les défis urbains et l'impact de la globalisation dans le contexte africain, gestion urbaine et municipale en Afrique. Dakar Sénégal, 17 pages.

Fotsing, E. et Assako Assako, R. J. (2007). Du numérique et de la gestion des villes Prolégomènes. (R. J. Assako Assako, Éd.)
Observatoires urbains et environnementaux en Afrique : des théories aux applications géomatiques, 14-28.

Huguenin-Richard, F. (2004). Diagnostiquer le risque routier en milieu urbain par SIG. Travail de Recherche, Montréal-Canada.

ONU-Habitat (2014). L'état des villes africaines Réinventer la transition urbaine. Naïrobi-Kenya: Programme des Nations Unies pour les Etablissements Humains (ONU-HABITAT) 2014. Récupéré sur http://www.unhabitat.org, 278 pages.

Ouedraogo, R. U. E. (2010). La gestion urbaine dans les pays du Sud. Mémoire de DEA en Géographie, Burkina Faso : Université de Ouagadougou. www.memoireonline.com.

Petnga Nyamen, S. P. (2018). Analyse diachronique de l'urbanisation de Garona: acteurs et marqueurs spatiaux. Thèse de Doctorat/Ph.D, Université de Ngaoundéré, Département de Géographie, Ngaoundéré, 396 pages.

Pumain, D. (2008). Articles pour le Dictionnaire La ville et l'urbain. Manuscrit auteur. Récupéré sur https://halshs.archives-ouvertes.fr/halshs00266515/document, 132 pages.

Tchotsoua, M., Briltey Bakulay, Bitjoka, L., Mohamadou, G., et Ayissi Eteme, A. (2001). Outils logiciels et cartographie géoréférencée : cas de l'observatoire de l'environnement urbain de Ngaoundéré. Workshop de Douala, 89-101.

Tchotsoua, M., et Bonvallot, J. (2000). L'érosion urbaine au Cameroun: processus, causes et stratégies de lutte. Réseau érosion, 20(1), 324-331. 